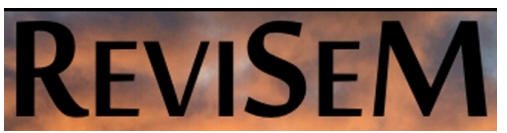

\title{
ALGUNS ESPAÇOS MÉTRICOS COMPLETOS NA TEORIA DE EQUAÇÕES DIFERENCIAIS PARCIAIS
}

\author{
Daniela Mota Teixeira \\ DMAI/Universidade Federal de Sergipe \\ danymota2010@gmail.com
}

\section{Resumo}

Neste artigo mostraremos a completude de alguns espaços importantes no estudo de equações diferenciais.

\section{Abstract}

In this article we will prove the completeness of some important spaces in the study of differential equations.

\section{Introdução}

A elaboração deste texto foi motivada a partir da diversidade de espaços métricos reconhecidos como completos e que aparecem na teoria das equações diferenciais, tanto clássica quanto moderna.

Dentre as aplicações mais importantes da teoria de espaços métricos em equações diferenciais está o teorema do ponto fixo de Banach. Com efeito, dado um problema de valor inicial do tipo

$$
\left\{\begin{array}{c}
\varphi^{\prime}(t)=f(t, \varphi(t)), \\
\varphi(0)=x_{0},
\end{array}\right.
$$

podemos transformá-lo em uma equação integral da seguinte forma

$$
\varphi(t)=x_{0}+\int_{0}^{t} f(s, \varphi(s)) d s .
$$

Para determinar a extistência de (1.2), recorremos a algum teorema de ponto fixo, mais precisamente o teorema do ponto fixo de Banach. Para isso, é definido um operador

$$
F: \mathcal{C}(I ; B) \longrightarrow \mathcal{C}(I ; B)
$$

onde o conjunto $\mathcal{C}(I ; B)$ é o espaço de aplicações contínuas $\varphi: I \longrightarrow B$. Mas para aplicar o teorema do ponto fixo de Banach, $\mathcal{C}(I ; B)$ deve ser um espaço completo. 
Teixeira, D. M.

Em meio aos muitos trabalhos referentes às aplicações dos espaços presentes neste texto, é interessante citar alguns destes, como os trabalhos de Andrade e Viana [2], Arrieta e Carvalho [1] e Nishiguchi [3]. Dentre os espaços estudados nesse texto, um deles é o $X_{\beta}$, caracterizado do seguinte modo

$$
X_{\beta}=\left\{u \in \mathcal{C}((0, T] ; \mathbb{E}):\|u\|_{X_{\beta}}=\sup _{t \in(0, T]} t^{\beta}\|u(t)\|_{\mathbb{E}}<+\infty\right\}
$$

tal que $\beta>0$ em que o espaço é um conjunto de aplicações contínuas em $\mathcal{C}((0, T] ; \mathbb{E})$, no qual $\mathbb{E}$ é um espaço de Banach.

Em [2], $X_{\beta}$ é definido com $\mathbb{E}=L^{r}\left(\mathbb{R}^{n}\right)$, porém não é mostrado que tal espaço é de Banach. Neste texto, mostraremos que $X_{\beta}$ é, de fato, um espaço de Banach.

Já em [1], $X_{\beta}$ é denotado por $K\left(\tau_{0}\right)$, em que o mesmo é um conjunto de aplicações contínuas em $\mathcal{C}\left(\left(0, \tau_{0}\right] ; X^{1+\epsilon}\right)$, onde $\epsilon>0$ e $X^{1+\epsilon}$ é um espaço de potências fracionárias de um operador e além disso um espaço de Banach. E o objetivo do texto é mostrar um teorema de existência e unicidade para equações do tipo $x^{\prime}=A x+f(t, y)$, resultado que pode ser aplicado a equações de Navier-Stokes e equações de calor.

Ao final, consideraremos o espaço $\mathcal{C}_{t_{0}, \phi_{0}}(b)$ (ver Definição 3.1) que é similar àquele tomado em [3], no qual é mostrado sua completude. O objetivo de [3] é estudar uma condição para solucionar problemas de valor inicial em equações diferenciais com retardo. Aqui, estamos interessados em dar detalhes da demonstração da Proposição 2.2 de [3].

\section{Conceitos Preliminares}

Nesta seção apresentaremos a notação básica utilizada no texto e alguns resultados do estudo de espaços métricos. Para conhecer as definições iniciais, consultar o livro Espaços Métricos [4]. Além disso, incluiremos algumas demonstrações para conveniência do leitor.

Denotaremos por $d$ a métrica e por $M$ o espaço métrico. Adotaremos a notação $\|\cdot\|$ para representar a norma de um espaço vetorial. Além disso, utilizaremos $\mathbb{E}$ para representar o espaço de Banach, que é um espaço vetorial normado e completo.

Proposição 2.1. Toda sequência convergente é de Cauchy. E sendo de Cauchy, é limitada.

Definição 2.2. Sejam $M$ e $N$ espaços métricos. Uma aplicação bijetiva $f: M \longrightarrow N$ tal que $d(f(x), f(y))=d(x, y)$ é denominada isometria.

Definição 2.3. Um homeomorfismo é uma aplicação bijetiva $f: M \longrightarrow N$ contínua, tal que, sua inversa $f^{-1}: N \longrightarrow M$ também é contínua. 
Teixeira, D. M.

Definição 2.4. Sejam $M, N$ espaços métricos. Uma aplicação $f: M \longrightarrow N$ é dita uniformemente contínua quando para todo $\epsilon>$, existe $\delta>0$ tal que

$$
d(x, y)<\delta \Rightarrow d(f(x), f(y))<\epsilon
$$

para quaisquer $x, y \in M$.

Definição 2.5. Um homeomorfismo uniforme é uma bijeção $g: M \longrightarrow N$ unirfomemnte contínua e sua inversa $g^{-1}: N \longrightarrow M$ também é.

Proposição 2.6. Seja $f: M \longrightarrow N$ um homeomorfismo uniforme. Uma sequência de pontos $\left(x_{n}\right)_{n \mathbb{N}}$ é de Cauchy se, e somente se, $\left(f\left(x_{n}\right)\right)_{n \in \mathbb{N}}$ é de Cauchy.

Demonstração. Seja $g: M \longrightarrow N$ um homeomorfismo uniforme e $\left(x_{n}\right)_{n \in \mathbb{N}}$ uma sequência de Cauchy em $M$. Pela Definição 2.4, dado $\epsilon>0$, existe $\delta>0$ tal que

$$
d(x, y)<\delta \Rightarrow d(f(x), f(y))<\epsilon
$$

para quaisquer $x, y \in M$. Mas, por hipótese, $\left(x_{n}\right)_{n \in \mathbb{N}}$ é de Cauchy, então dado $\delta>0$, existe $n_{0} \in \mathbb{N}$ tal que se $m, n>n_{0}$ então $d\left(x_{m}, x_{n}\right)<\delta$. Mas a continuidade uniforme implica que $d\left(f\left(x_{m}\right), f\left(x_{n}\right)\right)<\epsilon$. Logo $\left(f\left(x_{n}\right)\right)_{n \in \mathbb{N}}$ é de Cauchy.

Reciprocamente, seja $\left(f\left(x_{n}\right)\right)_{n \in \mathbb{N}}$ uma sequência de Cauchy em $N$. Mas, pela Definição $2.5, f^{-1}: N \longrightarrow M$ é uniformemente contínua, o que implica que $\left(x_{n}\right)_{n \in \mathbb{N}}$ é de Cauchy.

Observação 2.7. Um homemorfismo que é uma isometria é um homeomorfismo uniformemente contínuo. Além disso, dados dois espaços $M$ e $N$ tais $M$ é completo, então a completude de $M$ implica na completude de $N$.

Observação 2.8. A proposição 2.6 será útil na demonstração do Teorema 3.3.

Definição 2.9. O espaço métrico $M$ é completo quando toda sequência de Cauchy em $M$ é convergente.

Definição 2.10. Sejam $X$ um conjunto, $M$ um espaço métrico e $\alpha: X \rightarrow M$ uma aplicação. A notação $B_{\alpha}(X ; M)$ representa o conjunto das aplicações $f: X \rightarrow M$ tais que $d(f, \alpha)=\sup _{x \in X} d(f(x), \alpha(x))<\infty$, com a métrica da convergência uniforme.

Proposição 2.11. Se o espaço métrico $M$ é completo então $B_{\alpha}(X ; M)$ é completo, sejam quais forem $X$ e $\alpha: X \rightarrow M$. 
Teixeira, D. M.

Demonstração. Seja $\left(f_{n}\right)_{n \in \mathbb{N}}$ uma sequência de Cauchy em $B_{\alpha}(X ; M)$. Fixe $x \in X$ de maneira arbitrária, assim a sequência $\left(f_{n}(x)\right)_{n \in \mathbb{N}}$ é de Cauchy em $M$. Por $M$ ser completo, existe para cada $x \in X$, o limite desta sequência. Neste caso, $\lim f_{n}(x)=$ : $f(x) \in M$.

Como a sequência é de Cauchy, pela Proposição ??, esta é limitada; então existe $c>0$ tal que

$$
d\left(f_{n}(x), \alpha(x)\right) \leq d\left(f_{n}, \alpha\right) \leq c
$$

para todo $n \in \mathbb{N}$ e todo $x \in X$. Fazendo $n \rightarrow \infty$ concluímos que $d(f(x), \alpha(x)) \leq c$ para todo $x \in X$. Logo $f \in B_{\alpha}(X ; M)$.

Agora, resta provar que $f_{n} \rightarrow f$ uniformemente em $X$. Então, dado $\epsilon>0$, existe $n_{0} \in \mathbb{N}$ tal que $m, n>n_{0} \Rightarrow d\left(f_{m}(x), f_{n}(x)\right)<\epsilon$ para qualquer $x \in X$, uma vez que $\left(f_{n}\right)_{n \in \mathbb{N}}$ é de Cauchy. Daí, fazendo $m \rightarrow \infty$ nesta desigualdade, concluímos que $n>n_{0} \Rightarrow d\left(f(x), f_{n}(x)\right)=d\left(f_{n}(x), f(x)\right) \leq \epsilon$ para todo $x \in X$. Portanto, $f_{n} \rightarrow f$ uniformemente, como queríamos demonstrar.

Proposição 2.12. Todo subespaço fechado de um espaço de Banach é Banach. Reciprocamente, todo subespaço de Banach de um espaço normado é fechado.

Demonstração. Seja $F \subset \mathbb{E}$ tal que $F$ é fechado. Tome $\left(x_{n}\right)_{n \in \mathbb{N}}$ de Cauchy em $F$. Mas, como $F \subset \mathbb{E}$ então $\left\{x_{n}\right\}_{n \in \mathbb{N}} \subset \mathbb{E}$, que por sua vez é completo, logo $x_{n} \mapsto x \in \mathbb{E}$, que implica que $x \in \bar{F}$. E como $F$ é fechado, temos que $x \in F$. Logo $\left(x_{n}\right)_{n \in \mathbb{N}}$ converge em $F$. Portanto $F$ é completo.

Reciprocamente, seja $F$ um subespaço de Banach de $\mathbb{E}$. Considere $y \in \bar{F}$, isto é, $y$ é aderente a $F$, então existe uma sequência $\left(y_{n}\right)_{n \in \mathbb{N}}$ em $F$ tal que $y_{n} \mapsto y$. Pela Proposição 2.1, podemos afirmar que $\left(y_{n}\right)_{n \in \mathbb{N}}$ é de Cauchy em $F$, e este é completo, logo $y \in F$. Portanto, $F$ é fechado.

\section{$3 \quad$ Resultados Principais}

Nesta seção será mostrada a completude de alguns espaços importantes no estudo de equações diferenciais. O estudo desses espaços motivaram a elaboração deste artigo e além disso, foram citados na introdução, alguns textos que se utilizaram de tais espaços.

Teorema 3.1. O conjunto $X_{\beta}$ é um espaço de Banach.

Demonstração. Inicialmente será verificado que a função proposta sobre $X_{\beta}$,

$$
\|u\|_{X_{\beta}}=\sup _{t \in(0, T]} t^{\beta}\|u(t)\|_{\mathbb{E}}
$$

ReviSeM, Ano 2018, No. 1, 50-58 
é de fato uma norma. Uma vez que, todo espaço normado torna-se um espaço métrico através da definição $d(x, y)=\|x-y\|$. Isto implica que a métrica provém da norma $\|\cdot\|$.

Sejam $u, v \in X_{\beta}$ e $\lambda \in \mathbb{R} \backslash\{0\}$ temos que

- $\|u\|_{X_{\beta}}=\sup _{t \in(0, T]} t^{\beta}\|u(t)\|_{\mathbb{E}} \neq 0$.

- $\operatorname{Como} \sup (\lambda f(x))=\lambda \sup (f(x))$, note que

$$
\begin{aligned}
\|\lambda u\|_{X_{\beta}} & =\sup _{t \in(0, T]} t^{\beta}\|\lambda u(t)\|_{\mathbb{E}} \\
& =|\lambda| \sup _{t \in(0, T]} t^{\beta}\|u(t)\|_{\mathbb{E}} \\
& =|\lambda|\|u\|_{X_{\beta}}
\end{aligned}
$$

- Uma vez que $\sup (f+g) \leq \sup f+\sup g$, podemos aplicar tal propriedade para obter

$$
\begin{aligned}
\|u+v\|_{X_{\beta}} & =\sup _{t \in(0, T]} t^{\beta}\|u(t)+v(t)\|_{\mathbb{E}} \\
& \leq \sup _{t \in(0, T]} t^{\beta}\left(\|u(t)\|_{\mathbb{E}}+\|v(t)\|_{\mathbb{E}}\right) \\
& \leq\|u\|_{X_{\beta}}+\|v\|_{X_{\beta}}
\end{aligned}
$$

Portanto, $\|u\|_{X_{\beta}}$ é uma norma e $X_{\beta}$ é um espaço métrico.

Agora, mostraremos que tal espaço é completo. Pela Definição 2.9, tome $\left(u_{n}\right)_{n \in \mathbb{N}} \in$ $X_{\beta}$ de Cauchy e portanto, $\sup _{t \in(0, T]} t^{\beta}\left\|u_{n}(t)\right\|<+\infty$. Posteriormente, defina $v_{n}(t)=$ $t^{\beta} u_{n}(t) \in B((0, T], \mathbb{E})$ e note que, dado $\epsilon>0$, existe $n_{0} \in \mathbb{N}$, com $m, n \geq n_{0}$, tal que

$$
\begin{aligned}
\sup _{t \in(0, T]}\left\|v_{n}(t)-v_{m}(t)\right\|_{\mathbb{E}} & =\sup _{t \in(0, T]}\left\|t^{\beta} u_{n}(t)-t^{\beta} u_{m}(t)\right\|_{\mathbb{E}} \\
& =\left\|u_{n}-u_{m}\right\|_{X_{\beta}}<\epsilon
\end{aligned}
$$

Logo, $\left(v_{n}\right)_{n \in \mathbb{N}}$ é de Cauchy em $B((0, T], \mathbb{E})$. Pela Proposição 2.11, pode-se afirmar que $B((0, T], \mathbb{E})$ é completo, uma vez que $\mathbb{E}$ por hipótese é um espaço de Banach e a função $\alpha$ fixada é a função identicamente nula. Daí, pela Definição 2.9 , temos que $\left(v_{n}\right)_{n \in \mathbb{N}}$ converge para $v \in B((0, T], \mathbb{E})$ tal que

$$
\sup _{t \in(0, T]}\left\|v_{n}(t)-v(t)\right\|_{\mathbb{E}} \longrightarrow 0
$$


Teixeira, D. M.

quando $n \rightarrow \infty$, isto é,

$$
\sup _{t \in(0, T]} t^{\beta}\left\|u_{n}(t)-t^{-\beta} v(t)\right\|_{\mathbb{E}} \longrightarrow 0, n \rightarrow \infty .
$$

É fácil ver que $u(t)=t^{-\beta} v(t) \in X_{\beta}$. De fato,

$$
\begin{aligned}
\|u\|_{X_{\beta}} & =\sup _{t \in(0, T]} t^{\beta}\|u(t)\|_{\mathbb{E}} \\
& =\sup _{t \in(0, T]} t^{\beta}\left\|t^{-\beta} v(t)\right\|_{\mathbb{E}} \\
& =\sup _{t \in(0, T]}\|v(t)\|_{\mathbb{E}}<+\infty .
\end{aligned}
$$

Logo, $u(t)=t^{-\beta} v(t) \in X_{\beta}$ e portanto $X_{\beta}$ é um espaço de Banach.

\subsection{Espaço $\mathcal{C}_{t_{0}, \phi_{0}}(b)$}

Esta seção é destinada ao estudo do espaço $\mathcal{C}_{t_{0}, \phi_{0}}(b)$. Assim, fixaremos algumas notações especiais.

Denotemos por $I$ o intervalo $I=[-r, 0]$ com $r>0$ e o conjunto de aplicações de $I$ em $\mathbb{E}$ contínuas, denotaremos por $\mathcal{C}(I, \mathbb{E})$. Considere ainda, a seguinte notação $\left[t_{0}, t_{0}+b\right]+I:=\left\{t+\theta: t \in\left[t_{0}, t_{0}+b\right], \theta \in I\right\}$.

Definição 3.2. Seja $\left(t_{0}, \phi_{0}\right) \in \mathbb{R} \times \mathcal{C}(I ; \mathbb{E})$ tal que

- Se $J=\left[t_{0}, t_{0}+b\right]$, para algum $b>0$. Dizemos que a aplicação $x: J+I \longrightarrow \mathbb{E} e ́$ uma continuação de $\left(t_{0}, \phi_{0}\right)$ sempre que $x_{t_{0}}=\phi_{0}$ e quando $x$ for contínua em $J$. Quando $t_{0}=0$, é dito simplesmente que $x$ é uma continuação de $\phi_{0}$. Além disso, se $x_{\mid J}$ é diferenciável, dizemos que $x$ é uma $C^{1}$-continuação.

- Para $b>0$, defina

$$
\mathcal{C}_{t_{0}, \phi_{0}}(b)=\left\{x \in \mathcal{C}(J+I, \mathbb{E}): x \text { é uma continuação de }\left(t_{0}, \phi_{0}\right)\right\}
$$

Chamamos o espaço $\mathcal{C}_{t_{0}, \phi_{0}}(b)$ como o espaço de continuação de comprimento $\left(t_{0}, \phi_{0}\right)$.

- Para $\phi \in \mathcal{C}(I, \mathbb{E})$ considere a continuação de $\phi$ do seguinte modo

$$
\bar{\phi}:= \begin{cases}\phi(s), & s \in I \\ \phi(0), & s \in \mathbb{R}_{+}\end{cases}
$$


Teixeira, D. M.

Teorema 3.3. Seja $\left(t_{0}, \phi_{0}\right) \in \mathbb{R} \times \mathcal{C}(I ; \mathbb{E})$. Para todo $b>0$, o espaço $\mathcal{C}_{t_{0}, \phi_{0}}(b)$ é um espaço métrico completo.

Demonstração. Considere o espaço $\mathcal{C}_{0,0}(b)$ e note que o conjunto é um espaço vetorial. Como todo $x \in \mathcal{C}_{0,0}(b)$ é contínuo e limitado, $\mathcal{C}_{0,0}(b)$ torna-se um espaço vetorial normado munido da métrica do supremo.

Defina o conjunto

$$
Y_{b}:=\{y \in \mathcal{C}([0, b], \mathbb{E}) ; y(0)=0\} .
$$

Note que $Y_{b} \subset \mathcal{C}([0, b], \mathbb{E})$ é fechado, mas o fato de $\mathcal{C}([0, b], \mathbb{E})$ ser um espaço de Banach, pela Proposição 2.12, $Y_{b}$ também é Banach.

Além disso, o fato de $\mathcal{C}_{0,0}(b)$ e $Y_{b}$ serem isometricamente homeomorfos implica que $\mathcal{C}_{0,0}(b)$ também é um espaço de Banach. Defina o operador $\mathcal{N}: \mathcal{C}_{t_{0}, \phi_{0}}(b) \longrightarrow \mathcal{C}_{0,0}(b)$, com $b>0$ e $s \in[0, b]+I$, por

$$
\mathcal{N} x(s)=x\left(t_{0}+s\right)-\bar{\phi}_{0}(s) .
$$

Inicialmente será mostrado que $\mathcal{N} x$ é contínuo. Temos que $t \mapsto x(t+\theta)$ é contínuo, $t \in\left[t_{0}, t_{0}+b\right]$. Logo, $s \mapsto x\left(t_{0}+s+\theta\right)$ é conínuo, $s \in[0, b]$. Então,

$$
s \mapsto \mathcal{N} x(s+\theta)=x\left(t_{0}+s+\theta\right)-\overline{\phi_{0}}(s+\theta)
$$

é contínuo, uma vez que $\phi_{0} \in \mathcal{C}(I ; \mathbb{E})$.

Agora, mostraremos que $\mathcal{N}$ está bem definido. Seja $x \in \mathcal{C}_{t_{0}, \phi_{0}}(b)$ e $\theta \in I$, note que

$$
(\mathcal{N} x)_{0}(\theta)=\mathcal{N} x(\theta)=x\left(t_{0}+\theta\right)-\overline{\phi_{0}}(\theta)=x_{t_{0}}(\theta)-\overline{\phi_{0}}(\theta)=\phi_{0}(\theta)-\phi(\theta)=0 .
$$

Logo, $\mathcal{N}$ está bem definido.

Além disso, será provado que $\mathcal{N}$ é bijetivo. Sejam $x, y \in \mathcal{C}_{t_{0}, \phi_{0}}(b)$ tais que $\mathcal{N} x=\mathcal{N} y$ daí,

$$
\begin{aligned}
\mathcal{N} x(s) & =\mathcal{N} y(s) \\
\Longleftrightarrow x\left(t_{0}+s\right)-\bar{\phi}_{0}(s) & =y\left(t_{0}+s\right)-\overline{\phi_{0}}(s) \\
\Longleftrightarrow x\left(t_{0}+s\right) & =y\left(t_{0}+s\right),
\end{aligned}
$$

para todo $s \in[0, b]+I$. O que implica que $x=y$ em $s \in\left[t_{0}, t_{0}+b\right]+I$. Portanto, $\mathcal{N}$ é injetivo.

Considere $y \in \mathcal{C}_{0,0}(b)$, isto é, $y:[0, b]+I \longrightarrow \mathbb{E}$ tal que $y_{0}(\theta)=0$ para todo $\theta \in I$ e $t \mapsto y(t+\theta)$, com $t \in[0, b]$ contínuo. Considere $x \in \mathcal{C}_{t_{0}, \phi_{0}}(b)$ de modo que $x\left(t_{0}+t+\theta\right)=y(t+\theta)+\overline{\phi_{0}}(t+\theta)$ daí,

$$
\mathcal{N}(t+\theta)=x(t-0+t+\theta)-\overline{\phi_{0}}(t+\theta)=y(t+\theta),
$$


Teixeira, D. M.

$\theta \in I$. O que implica que $y=\mathcal{N} x$. Logo, $\mathcal{N}$ é sobrejetivo.

Por último, mostraremos que o operador $\mathcal{N}$ é de fato uma isometria, isto é,

$$
\begin{aligned}
\|\mathcal{N} x-\mathcal{N} y\| & =\sup _{s \in[0, b]+I}\left\|x\left(t_{0}+s\right)-\overline{\phi_{0}}(s)-y\left(t_{0}+s\right)+\overline{\phi_{0}}(s)\right\| \\
& =\sup _{s \in[0, b]+I}\left\|x\left(t_{0}+s\right)-y\left(t_{0}+s\right)\right\| \\
& =\|x-y\| .
\end{aligned}
$$

com $x, y \in \mathcal{C}_{t_{0}, \phi_{0}}(b)$. Logo, $\mathcal{C}_{t_{0}, \phi_{0}}(b)$ é um isometricamente homeomorfo a $\mathcal{C}_{0,0}(b)$. Portanto, pela Proposição $2.6, \mathcal{C}_{t_{0}, \phi_{0}}(b)$ é completo. 
Teixeira, D. M.

\section{Referências}

[1] Arrieta, José M.; Carvalho, Alexandre N.: Abstract parabolic problems with critical nonlinearities and applications to Navier-Stokes and heat equations. Trans. Amer. Math. Soc. 352 (2000), no. 1, 285-310.

[2] de Andrade, Bruno; Viana, Arlúcio: On a fractional reaction-diffusion equation, Z. Angew. Math. Phys. 68 (2017), no. 3, Art. 59, 11 pp.

[3] J. Nishiguchi, A necessary and sufficient condition for well-posedness of initial value problems of retarded functional differential equations, J. Differential Equations 263 (2017), no. 6, 3491-3532.

[4] LIMA, Elon L. Espaços Métricos, Elon Lages Lima. 4.ed. Rio de Janeiro: IMPA, 2009. 